\title{
CALIBRAÇÃO E DESEMPENHO DO ABRIGO METEOROLÓGICO DE BAIXO CUSTO EM CONDIÇÕES DE CAMPO
}

\author{
BARATTO, Jakeline - jekeline.jake@hotmail.com \\ Universidade Federal de Santa Maria / UFSM
}

\author{
GALVANI, Emerson - egalvani@usp.br \\ Universidade de São Paulo / USP
}

\author{
WOLLMANN, Cássio Arthur - cassio_geo@yahoo.com.br \\ Universidade Federal de Santa Maria / UFSM
}

\begin{abstract}
RESUMO: O objetivo deste trabalho foi instalar, calibrar e avaliar o desempenho de abrigos meteorológicos de baixo custo, em condições de campo, para medições de temperatura e umidade relativa do ar. Para esta pesquisa foram utilizados dados de temperatura e umidade relativa do ar do abrigo meteorológico de baixo custo $(A B C)$ e dos dados da estação meteorológica automática móvel (EMA), ambos instalados na bacia hidrográfica do Rio do Boi na divisa entre os estados de RS e SC. O período utilizado para essa avaliação foi o mês de agosto de 2017. Utilizou-se os seguintes parâmetros estatísticos para a análise dos dados: média, mínimo, máximo, amplitude, desvio médio e desvio padrão. O desempenho dos modelos foi avaliado em função dos coeficientes de correlação (R) e determinação (R2). Os resultados revelaram que o abrigo $A B C$ se mostrou eficiente para os dados de temperatura e umidade relativa do ar. Assim, os valores encontrados estão dentro do erro previsto $\left(0,3^{\circ} \mathrm{C}\right.$ para a temperatura do ar e $1,7 \%$ para a umidade relativa) no manual de instruções do sensor, apesar de valores diferenciados entre eles nos períodos de aquecimento e resfriamento para os dados amostrados. Dessa forma, 0 abrigo $A B C$ se mostrou eficiente quanto a temperatura e umidade relativa do ar, para o período analisado e em condições de campo.
\end{abstract}

PALAVRAS-CHAVE: temperatura do ar; umidade relativa do ar; abrigo meteorológico

\section{CALIBRATION AND PERFORMANCE OF LOW COST METEOROLOGICAL SHIELD IN FIELD CONDITIONS}

ABSTRACT: This research concerns in to install, calibrate and evaluate the performance of low cost meteorological shield, in natural and outside conditions, for temperature and relative humidity measurement. Was used the air temperature and relative humidity data of the low meteorological shield (ABC) and the automatic meteorological station (EMA), both installed at "Rio do Boi" watershed. The period used for evaluation was August 2017. Were used the following statistical parameters for data analysis: mean, maximum, minimum, amplitude, mean deviation and standard deviation. The performance of the models was evaluated according to the correlation (R) and determination (R2) coefficients. Results showed that the meteorological shield $A B C$ was efficient to air temperature and relative humidity data. The values showed that the data are within the error predicted by the instrumental instructions, although the values were different between the data in the period of heating and cooling. Therefore, the meteorological shield $A B C$ was efficient for air temperature and relative humidity data in the analyzed interval under field conditions

KEYWORDS: Air temperature; relative humidity; meteorological shield.

\section{INTRODUÇÃO}

As pesquisas climatológicas utilizam-se de instrumentos meteorológicos de baixo custo para a obtenção de dados dos atributos climáticos, principalmente em locais onde não há abrangência da rede meteorológica oficial 
e em locais remotos. Outro fator de importante impacto é o alto custo das estações meteorológicas automáticas, conforme destacam Torres et al., (2015). Diante desse cenário, os abrigos meteorológicos, também chamados de escudos de radiação, vêm sendo o foco de diversas pesquisas sobre a sua precisão na medição da temperatura do ar (ROJAS; GILETE; MASON, 2014; TANARA; HOHEISEL, 2007; ARMANI; GALVANI, 2006; SERAFINI JÚNIOR; ALVES, 2014; ALVES; SERAFINI JÚNIOR, 2013).

Os abrigos meteorológicos são utilizados como uma blindagem contra a radiação de onda curta, chuva e intemperes (OMM, 2012). Nesse sentido, Yang et al., (2016) colocam que, devido ao sensor e a estrutura do abrigo, podem ocorrer erros de medição de até $0,8^{\circ} \mathrm{C}$. Assim, o fluxo do ar, o regime de radiação e o revestimento do material influenciam sobre o erro de medição da temperatura do ar. Corroborando com os autores, Bernard et al., (2019) também ressaltam que o abrigo é usado como um escudo para proteção do sensor da radiação solar, e que isso introduz um erro na medição.

Na tentativa de confeccionar um abrigo meteorológico de baixo custo e com alto desempenho, vários autores dedicaram-se a essa pesquisa com diferentes materiais e modelos. Os materiais mais usados na confecção de abrigos são: madeira (SERAFINI JÚNIOR, 2010; ROJAS; GILETE; MAZON, 2014), PVC (AZEVEDO; TARIFA, 2001; CASTELHANO; ROSEGHINI, 2011, LOPES; JARDIM, 2012; FIALHO et al., 2018) e chapa de ferro galvanizado (ARMAN; GALVANI, 2006; HOPPE, et al., 2015). Outros materiais mais modernos também estão sendo empregados para confeccionar modelos como o uso de impressora 3D, sendo utilizado o material PLA (filamento termoplástico de Poliácido Lático) (SILVA et al., 2018).

Os modelos de abrigos meteorológicos mais usuais são, a tela de Stevenson (ROJAS; GILETE; MAZON, 2014; DOBRE; SETAN; MERLONE, 2018), o modelo de ventilação natural (HUBBARD; LIN, 2003; ARMANI; GALVANI, 2006) e o modelo de ventilação aspirado (TARARA; HOHEISEL, 20107; YANG et al., 2016). Assim, alguns autores vêm testando e comparando esses três modelos de abrigos, a fim de apresentar os que têm o menor erro na medição da temperatura do ar.Yang et al., (2016) analisaram os três modelos de abrigos meteorológicos para obter o melhor modelo, em função do erro de medição da temperatura do ar. Bernard et al., (2019) compararam 18 tipos de abrigos meteorológicos, entre os quais estão modelos de estações meteorológica automáticas (Gill), modelos oficiais de estação meteorológica convencional (tela de Stevenson) e modelos de ventilação forçada, chamado pelo autor de modelos híbridos.

Assim, Rojas, Gilete e Mazon (2014) avaliaram diferentes abrigos meteorológicos, com a finalidade de compor uma estação meteorológica de baixo custo para auxiliar no monitoramento climático em países em desenvolvimento. Fizeram simulações de abrigos considerando materiais e tamanhos diferentes com base na tela de Stevenson, modelo recomendado pela OMM (organização meteorológica mundial); sendo um tamanho igual ao recomendado pela OMM, outro de tamanho menor e dois outros modelos alternativos. A partir das simulações utilizando as propriedades físicas de cada material, os autores concluíram que o abrigo menor apresentou melhor desempenho. Outra conclusão dos autores foi em função do material, sendo o abrigo de madeira o que desempenhou os melhores resultados. 
Alves e Serafini (2013) e Serafini Júnior e Alves (2014) avaliaram estatisticamente três modelos de abrigos meteorológicos, o modelo TAR e o modelo 4AS, ambos de madeira, e o abrigo ABC construído com chapa de ferro galvanizada com um abrigo oficial. Para os dados de temperatura do ar (SERAFINI JÚNIOR; ALVES, 2014) os três modelos se mostraram satisfatórios com um alcance de $80 \%$ no valor dos dados. Em relação a umidade relativa do ar (ALVES; SERAFINI, 2013) os resultados, na comparação dos três modelos com o modelo oficial, também se mostraram satisfatórios.

Diferentes modelos com PVC foram testados por Valin Jr et al., (2015). Esses autores testaram cinco modelos de abrigos meteorológicos, sendo eles dois modelos feitos com tubo de PVC, um vertical e outro horizontal, um modelo com prato de plásticos, outro com um pote branco e o ultimo de madeira. Esses abrigos foram utilizados na comparação com uma estação meteorológica da Fazenda Experimental da Universidade Federal de Mato Grosso. Os resultados mostraram que os abrigos do tipo vertical e de pote não foram eficientes no desempenho para a coleta dos dados. Os demais se mostraram alternativas viáveis para a obtenção dos dados. Assim, os autores concluem que não apenas o material é importante para a construção dos abrigos, mas também a arquitetura deles.

Armani e Galvani (2006) ressaltam a inviabilidade de abrigos meteorológicos que utilizam baterias ou pilhas para o seu funcionamento, o que vem tornar o custo muito alto, impossibilitando a pesquisa. Outro aspecto levantado pelos autores é de que abrigos alimentados por energia elétrica, como os de ventilação forçada, dificultam a expansão da pesquisa, sendo que em muitos locais experimentais não se tem energia disponível., e assim, dificulta a expansão da pesquisa. Os abrigos de madeira também têm as suas implicações devido, muitas vezes, ao seu tamanho e peso que dificultam o seu transporte e instalação em lugares remotos (CASTELHANO; ROSEGHINI, 2011)

O modelo do abrigo meteorológico $A B C$ proposto por Armani e Galvani (2006) foi baseado no modelo de no modelo 41003 10-Plate Gill Radiation Shield da Campbell e usa sistema de ventilação passiva. Esse modelo vem sendo testado e utilizado por diversos autores em pesquisa de climatologia geográfica (HOPPE et al., 2015; ALVES; SERAFINI JÚNIOR, 2013; SERAFINI JÚNIOR; ALVES, 2014; GALVANI; LIMA, 2006; GALVANI; LIMA; FALCÃO,2010; LIMA; GALVANI; FALCÃO,2010; SANTOS; MORAIS; GALVANI, 2015; WOLLMANN; SIMIONI (2013), SIMIONI; WOLLMANN (2015, 2016) e WOLLMANN; SIMIONE; IENSSE (2016). Dessa forma, esse modelo de abrigo meteorológico vem sendo consolidado na pesquisa em climatologia devido a sua confiabilidade e precisão dos dados, tanto de temperatura do ar quanto de umidade relativa do ar. Outro fator de grande relevância é o baixo custo e a praticidade de transporte e instalação.

Nesse contexto, observa-se que o uso do abrigo meteorológico de baixo custo, modelo $A B C$, vem sendo utilizado nas pesquisas climatológicas a mais de uma década e contribuindo para o melhoramento das técnicas de coleta de dados climáticos. Assim, as pesquisas sobre o uso dos abrigos meteorológicos se dividem em dois cenários: pesquisas em ambientes urbanos que buscam entender o clima urbano a partir de dados locais de temperaturas e umidade do ar (LOPES; SILVA, 2012). O outro cenário é com análises topoclimáticas, onde os ambientes naturais são foco de estudos, em que os autores buscam 
compreender a variação da temperatura e umidade em relação a altitude e uso e cobertura da terra (GALVANI; LIMA, 2006; GALVANI et al., 2008). Diante disso, o objetivo desse trabalho foi instalar, calibrar e avaliar o desempenho de abrigos meteorológicos de baixo custo, em condições de campo, para medições de temperatura e umidade relativa do ar.

\section{MATERIAIS E MÉTODOS}

Depois de concedida a autorização de pesquisa, foi instalada uma estação meteorológica automática (EMA) na bacia hidrográfica do Rio do Boi (BHRB). Precisou-se de autorização devido à área de estudo estar inserida, grande parte, no Parque Nacional de Aparados da Serra (PNAS). O PNAS foi implementado no ano de 1959 pelo Decreto Estadual no 47.446, alterado em 17/03/1972 pelo Decreto Federal no 70.296. O parque possui uma área de 10.250 ha. O limite do parque ao Norte é a estrada que liga Cambará do Sul a Praia Grande (RS-429) e a Oeste o rio Camisas.

A BHRB tem uma área de aproximadamente de $73,1 \mathrm{~km}^{2}$. No entanto, utilizou-se apenas a o setor da bacia hidrográfica que abrange o cânion Itaimbezinho, que foi monitorada pelos equipamentos. Essa porção da bacia hidrográfica tem área de $57,3 \mathrm{~km}^{2}$. Assim, na figura 1 , observa-se a localização do PNAS, bem como, a localização da EMA instalada

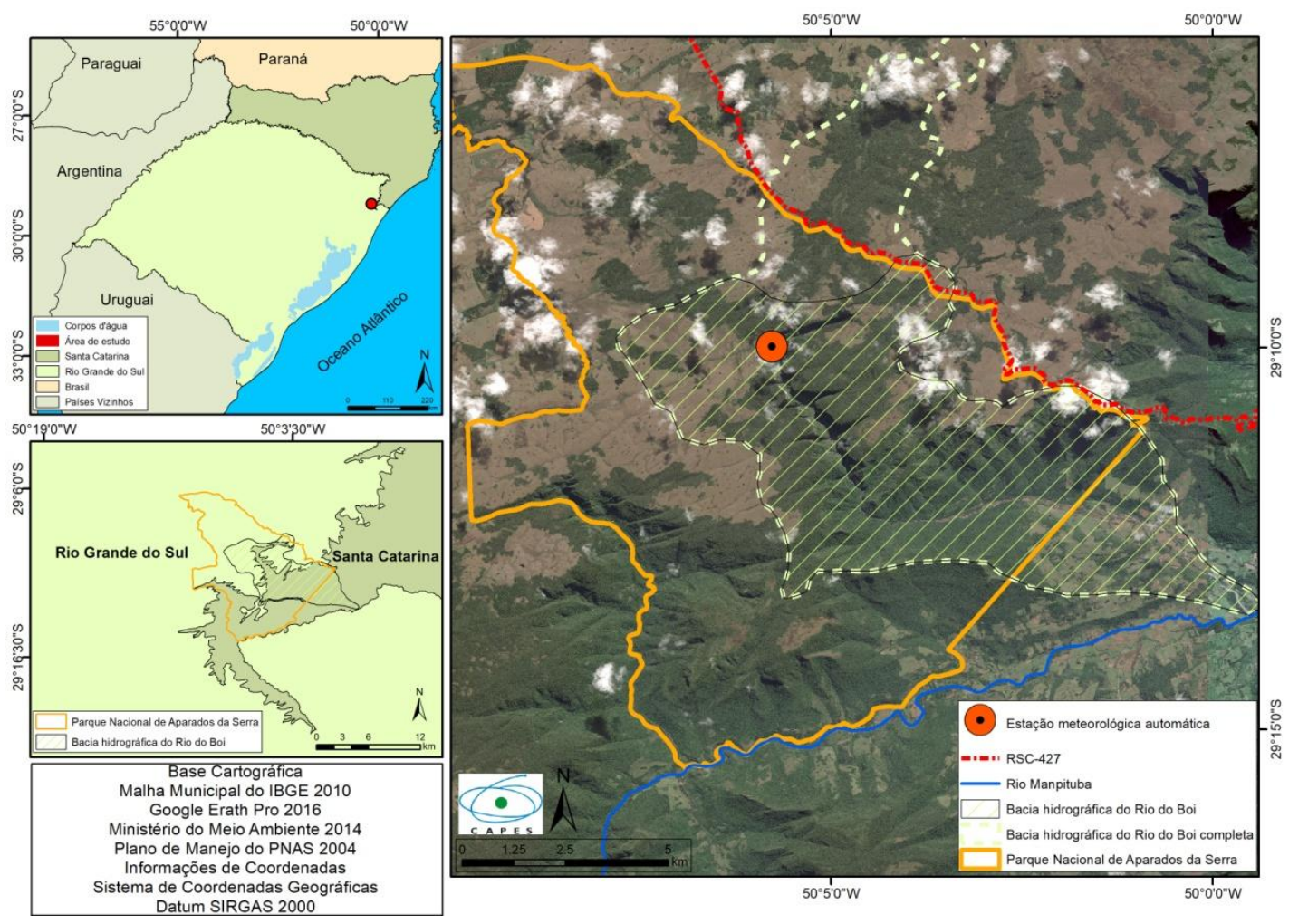

Figura 1 - Localização do Parque Nacional de Aparados da Serra.

A EMA modelo Campbell CR 1000 foi instalada nas coordenadas $587837.17 \mathrm{mE}$ e $677228.26 \mathrm{mS}$, fuso 22 e a uma altitude de $930 \mathrm{~m}$. A EMA pertence ao Laboratório de Climatologia e Biogeografia da Universidade de São 
Paulo, sendo instalada no dia $11 / 07 / 2017$ no parque. Os sensores de temperatura e umidade relativa do ar são do modelo HMP35C e foram devidamente calibrados em câmara selada com isolação térmica (GOBO, 2018).

Diante disso, juntamente com a EMA foi instalado um abrigo meteorológico de baixo custo (Abrigo $A B C$ ), que foi construído conforme modelo proposto por Armani e Galvani (2006) contendo um datalogger modelo HT 500. $O$ abrigo $A B C$ foi construído com chapa de ferro galvanizado de $0,5 \mathrm{~mm}$ de espessura, cujo modelo é inspirado ao 41003 10-Plate Gill Radiation Shield da Campbell (ARMANI; GALVANI, 2006). A estrutura do abrigo era de $11,5 \mathrm{~cm}$ de altura e $18 \mathrm{~cm}$ de diâmetro e, posteriormente a construção, foi pintada na cor branca (HOPPE, et al.,2015).

Abrigos meteorológicos de baixos custos foram construídos para essa pesquisa, no entanto, o datalogger já havia sido utilizado em outra pesquisa. Ambos eram pertencentes ao Laboratório de Climatologia Ambiental e Subtropical (LaCAS), do Departamento de Geociências da Universidade Federal de Santa Maria. O termo-higrômetro eletrônico de marca Instrutherm, modelo HT500 foi utilizado para a coleta dos dados necessários. Conforme Milanesi (2017), a precisão do datalogger HT500 com relação a temperatura do ar é de $+/-1,0^{\circ} \mathrm{C}$ e a escala de funcionamento é de $-40{ }^{\circ} \mathrm{C}$ a $70^{\circ} \mathrm{C}$. Em relação a umidade relativa do ar a precisão é de aproximadamente $+/-3 \%$, sendo que a escala é de 0 a $100 \%$.

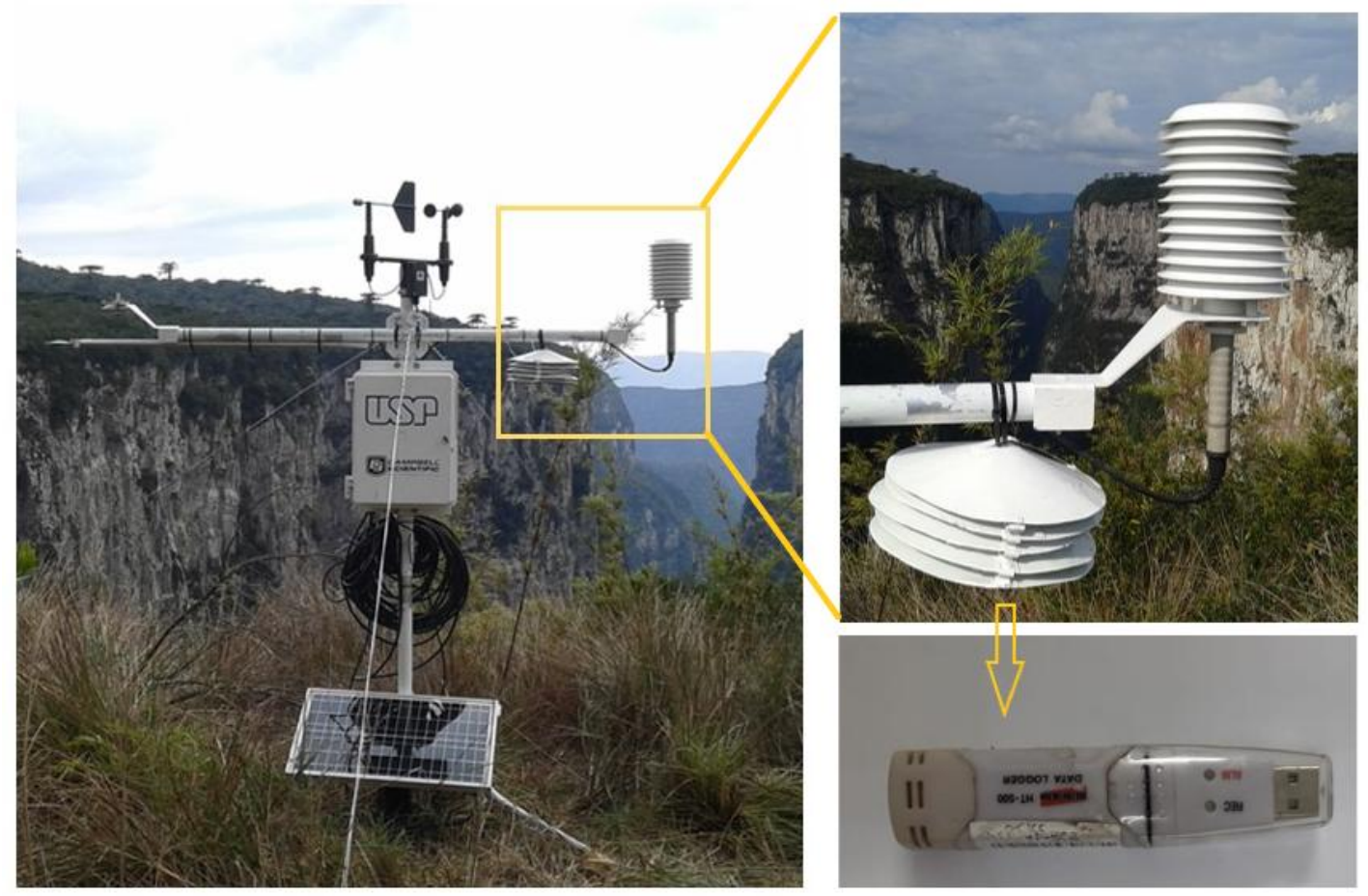

Figura 2 - Estação meteorológica automática e abrigo meteorológico instalado em campo.

A EMA foi programada para a coleta dos dados no intervalo de 10 minutos, já o sensor HT 500 foi programado para a coleta dos dados horários. Assim, os dados selecionados para essa pesquisa foram de hora em hora, logo se utilizou dos registros da EMA também a cada hora. O período de amostragem 
para a avaliação do abrigo $A B C$ foi de 01/08/2017 a 31/08/2017, totalizando dois pares de dados de temperatura do ar com 744 registros e igual quantidade para a umidade relativa do ar.

Durante o período de calibragem não foram observadas falhas nos dados, o que vem a mostrar a qualidade dos abrigos perante ao tempo de uso. Jardim (2018) ressalta que não há um limite de dias ou horas recomendado para o processo de calibração de um abrigo meteorológico de baixo custo. Porém, intervalos maiores possibilitam que abrigo fique exposto às condições adversas de tempo, o que vem a implicar na ampliação da margem de verificação e também na correção junto aos dados da estação meteorológica oficial.

A avaliação do abrigo meteorológico ocorreu conforme o apresentado por Armani e Galvani (2006). Assim, foram utilizados os parâmetros estatísticos como: máximo, mínimo, média aritmética simples, desvio médio, e desvio padrão sem distinções horárias e diárias. E ainda os parâmetros de regressão linear e de coeficientes de determinação, para a relação da temperatura e umidade relativa do ar dos dados do $A B C$ para com os dados da EMA. $O$ desempenho dos modelos foi avaliado em função do coeficiente de correlação (R) e determinação (R2).

\section{RESULTADOS E DISCUSSÕES}

\subsubsection{TEMPERATURA DO AR}

Os dados de temperatura do ar, registrados no abrigo meteorológico $A B C$ (Tabc) apresentaram uma diferença média da temperatura de $0,3^{\circ} \mathrm{C}$ superior aos obtidos junto a EMA (Tema). Armani e Galvani (2006) encontraram valores similares da ordem de 0,2oC. Porém Hoppe et al., (2015), ao calibrarem os dados do abrigo ABC com a Estação meteorológica convencional da INMET, instalada na Universidade Federal de Santa Maria, encontraram uma diferença de $1,3^{\circ} \mathrm{C}$ entre os dois sensores, cerca de $1,1^{\circ} \mathrm{C}$ a mais que Armani e Galvani (2006). No entanto, Serafini Júnior e Alves (2014) testaram o miniabrigo meteorológico $A B C$ para os períodos sazonais e encontraram para o inverno uma amplitude térmica de $0,4^{\circ} \mathrm{C}$. Com isso, observa-se que os resultados do equipamento avaliado corroboram com o apresentado e testado pela literatura.

A diferença entre as amplitudes térmicas identificadas foi de $1,8^{\circ} \mathrm{C}$ corroborando com o encontrado por Armani e Galvani (2006), em que a diferença entre as amplitudes foi de $1,9^{\circ} \mathrm{C}$. Entretanto, Hoppe et al. (2015) encontraram uma amplitude menor entre os dois de $0,5^{\circ} \mathrm{C}$. Essas diferenças encontradas pelos autores são muito mais abrangentes, tendo em vista que podem estar associadas a diferentes períodos do ano, o que vem a implicar em diferentes cargas térmicas sobre o abrigo meteorológico. Outro fator de possível alteração nas diferenças entre os autores é o achatamento dos abrigos meteorológicos, implicando nas condições de ventilação e transferência de calor no interior do abrigo (JARDIM, 2018). Na tabela 1, observam-se os resultados dos parâmetros estatísticos para ambos os abrigos meteorológicos. 
Tabela 1 - Parâmetros estatísticos elementares, em ${ }^{\circ} \mathrm{C}$, para a aferição dos dados obtidos nos abrigos denominados de $A B C$ e a EMA para período de 01/08/2017 a 31/08/17.

\begin{tabular}{lccc}
\hline Parâmetros & T ABC $\left.^{\circ}{ }^{\circ} \mathbf{C}\right)$ & T EMA $\left({ }^{\circ} \mathbf{C}\right)$ & T ABC - T EMA \\
\hline Média & 12,6 & 12,3 & 0,3 \\
\hline Mínimo & $-1,1$ & $-1,0$ & $-0,1$ \\
\hline Máximo & 30,4 & 28,6 & 1,8 \\
\hline Amplitude & 31,5 & 29,6 & 1,9 \\
\hline Desvio médio & 4,4 & 3,8 & 0,6 \\
\hline Desvio padrão & 5,7 & 5,1 & 0,7 \\
\hline
\end{tabular}

T ABC: Temperatura do ar do abrigo meteorológico ABC;

T EMA: Temperatura do ar da estação meteorológica;

T ABC - T EMA: Diferença entre a temperatura do ar do ABC e EMA.

Fonte: Trabalho de campo (2017).

Serafini Júnior e Alves (2014) apontam que conforme as amplitudes térmicas forem menores entre os abrigos em comparação, maior será a eficiência do abrigo meteorológico. Nesse contexto, a diferença entre as temperaturas mínimas dos dois abrigos foi de $-0,1^{\circ} \mathrm{C}$. Isso indica que, para as temperaturas mínimas, o $A B C$ apresentou as temperaturas mais baixas que a EMA. Com relação à temperatura máxima, a diferença foi de $1,8^{\circ} \mathrm{C}$. Isso ocorre porque, segundo Armani e Galvani (2006), nos horários de maior insolação e aquecimento há o aumento da temperatura do ar dentro do abrigo, que é decorrente da emissão de ondas longas pela chapa de metal que compõe o abrigo. Hoppe et al., (2015) também encontraram as maiores diferenças entre as temperaturas máximas.

Nesse sentido, Yang et al., (2016) destacam que a baixa velocidade do vento associada a alta radiação podem causar erros na medição de temperatura do ar. Diante disso, a autora ainda ressalta que a circulação de ar dentro do abrigo meteorológico é importante para evitar os erros de medição e o tempo de resposta do abrigo. Bernad et al., (2019) também corrobora com os autores acima citados, e ressaltam que as maiores diferenças entre as temperaturas registradas nos diferentes abrigos meteorológicos podem ser causadas pela baixa velocidade do vento.

A diferença entre o desvio padrão foi de $0,7^{\circ} \mathrm{C}$, evidenciando que $\mathrm{O}$ abrigo $A B C$ tem maior desvio entre os registros de temperatura do ar, o que influencia na precisão das informações de temperatura registradas no $A B C$. Diante disso, na análise geral dos dados de temperatura do ar observou-se que a Tabc, na maioria do período, ficou acima da Tema. Porém, quando é registrada a temperatura mínima nota-se que a Tabc diminui mais acentuadamente que a Tema.

A amplitude térmica mensal para o abrigo $A B C$ foi de $31,5^{\circ} \mathrm{C}$ e de 29,6 ${ }^{\circ} \mathrm{C}$ para o da EMA. Essa diferença mostra que no $A B C$ a temperatura média mensal foi de $1,9^{\circ} \mathrm{C}$. Dessa forma, observaram-se períodos com temperatura 
negativa, sendo que o mínimo registrado no abrigo $A B C$ foi de $-1,1^{\circ} \mathrm{C}$ e $\mathrm{o}$ máximo foi de $30,4^{\circ} \mathrm{C}$ (Figura 3 ).

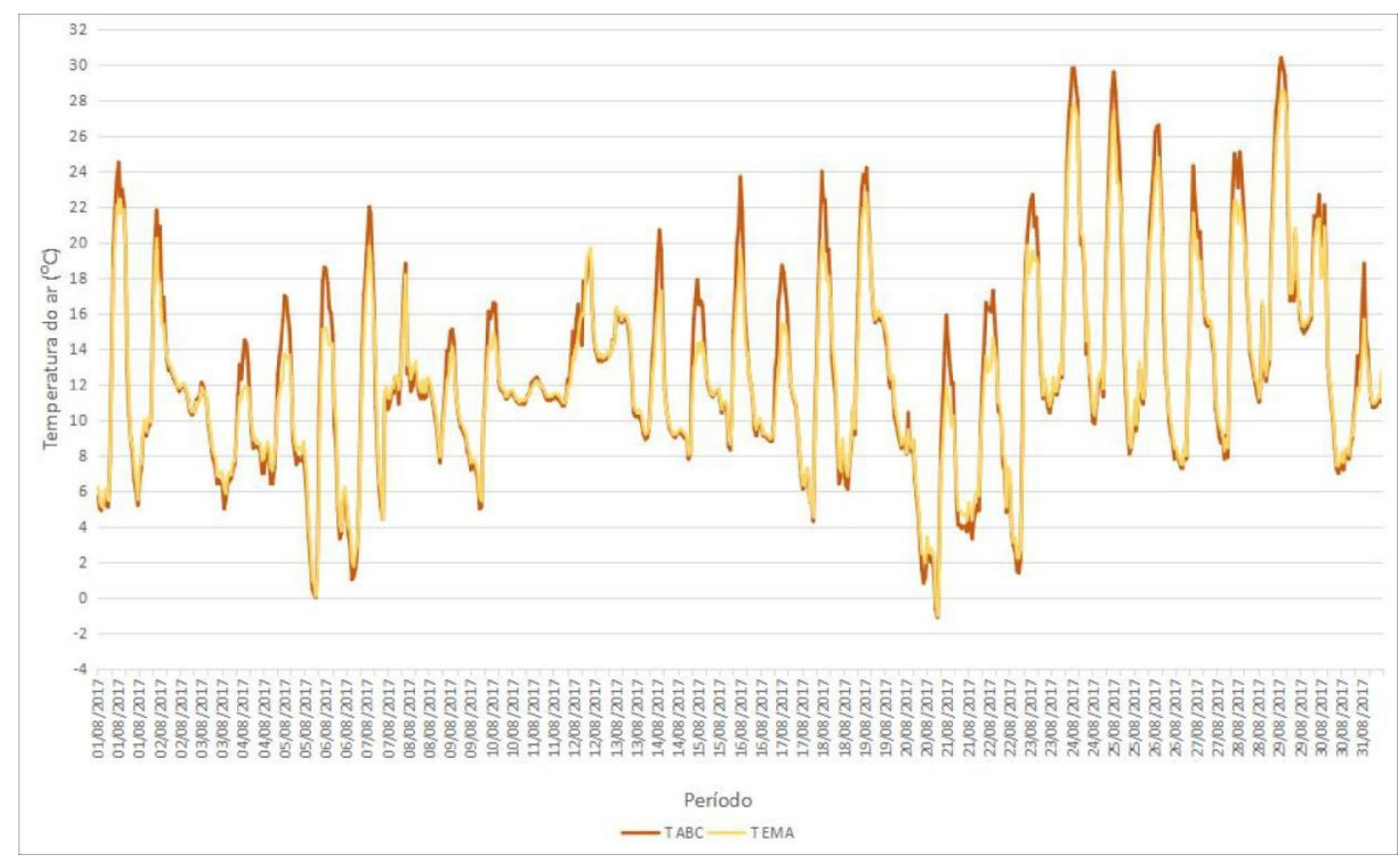

Figura 3 - Valores registrados para a média horária da temperatura do ar $\left({ }^{\circ} \mathrm{C}\right)$ da EMA e do $A B C$ para o período o mês de agosto de 2017.

Ao comparar a média da temperatura do ar diária entre as temperaturas dos dois abrigos, observa-se que no período de insolação, das 07h00min horas até $15 \mathrm{~h} 00 \mathrm{~min}$ horas, a temperatura do abrigo $A B C$ é mais elevada que a temperatura da EMA. Após as $15 \mathrm{~h} 00 \mathrm{~min}$ horas, a temperatura do ar registrada no $A B C$ é menor que a registrada na EMA. Armani e Galvani (2006) salientam que nos horários de maior resfriamento, por ser mais ventilado e aberto do que abrigo meteorológico oficial encontrado na EMA, $O$ abrigo $A B C$ diminui $a$ temperatura do ar dentro do abrigo mais rapidamente.

Os desvios de temperatura entre os dois abrigos são maiores no período de aquecimento, apresentando o máximo de desvio às $12 \mathrm{~h} 00 \mathrm{~min}$, ou seja, no período em que ocorre o aquecimento do ABC. Nessa perspectiva, Yang et al., (2016) colocam que o maior erro de medição da temperatura do ar é no período de maior fluxo de radiação solar. Os autores também colocam que a altitude é um importante fator no erro de medição. Nesse contexto, observou-se que nesse período o máximo desvio entre os dois abrigos foi de $2,5^{\circ} \mathrm{C}$. Então justifica-se essa elevada diferença conforme o relatado por Armani e Galvani (2006): pela absorção da radiação direta, o que implica na maior concentração de calor dentro do abrigo.

No período de resfriamento (17h00min até 06h00min), o desvio da temperatura é negativo. Isto é, o abrigo $A B C$ tem temperaturas, em média, $0,5^{\circ} \mathrm{C}$ abaixo que a do abrigo da EMA, ao contrário do período de aquecimento, corroborando com o encontrado por Jardim (2018). Na figura 4, observa-se a variação da temperatura do ar nos dois abrigos e o desvio entre eles 


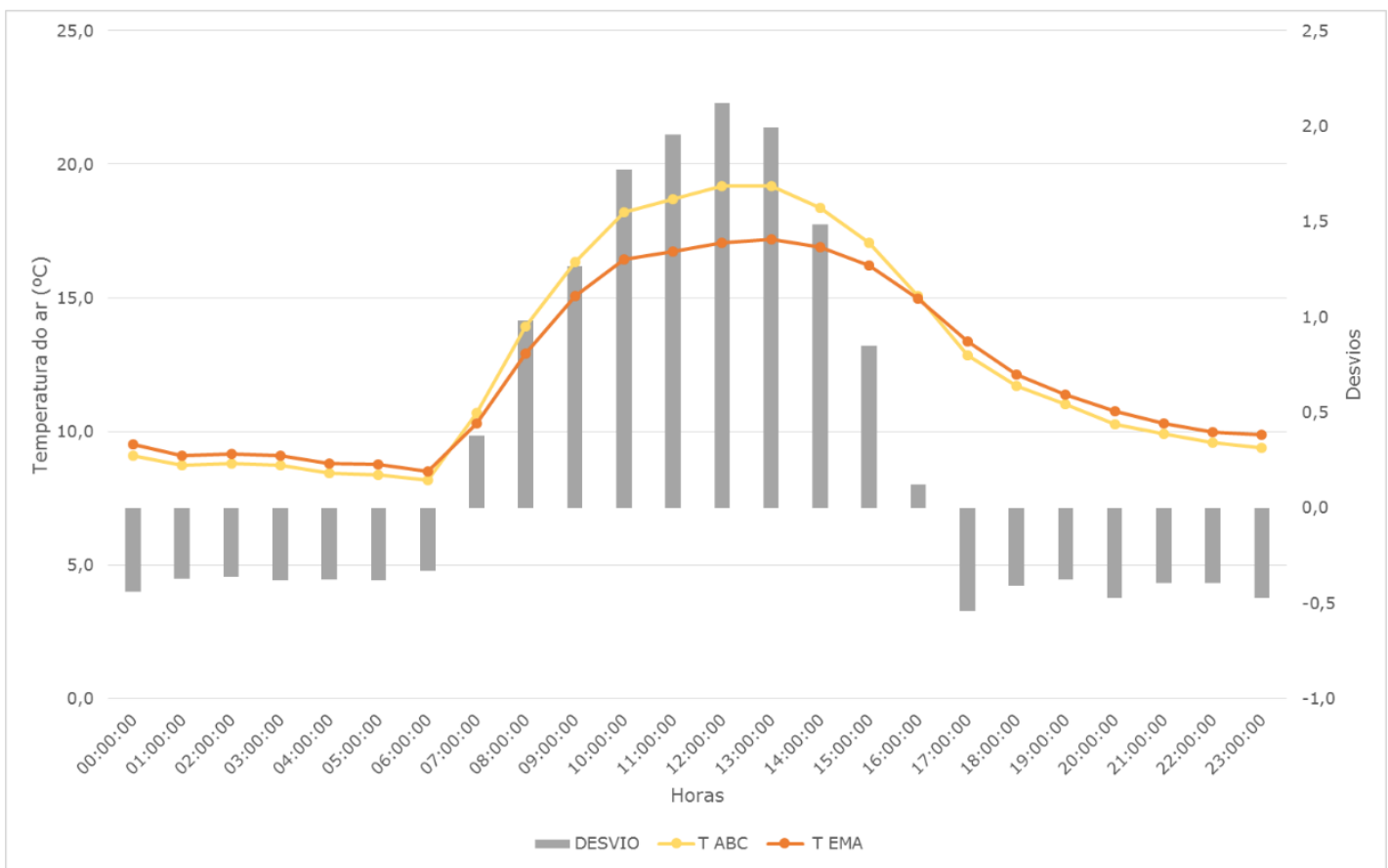

Figura 4 - Valores registrados para a média horária da temperatura do ar $\left({ }^{\circ} \mathrm{C}\right)$ da EMA e do $A B C$ para o período analisado, e o desvio absoluto entre os dois abrigos meteorológicos $\left({ }^{\circ} \mathrm{C}\right)$.

O coeficiente de correlação entre as duas variáveis, a temperatura medida pela EMA e a temperatura medida pelo $A B C$, foi de 0,99. Dessa forma, apresenta-se uma forte relação entre as duas variáveis. O coeficiente de determinação também se mostrou eficiente para os dados de temperatura do ar, sendo o $\mathrm{R}^{2}$ igual a 0,97 conforme a Figura 5.

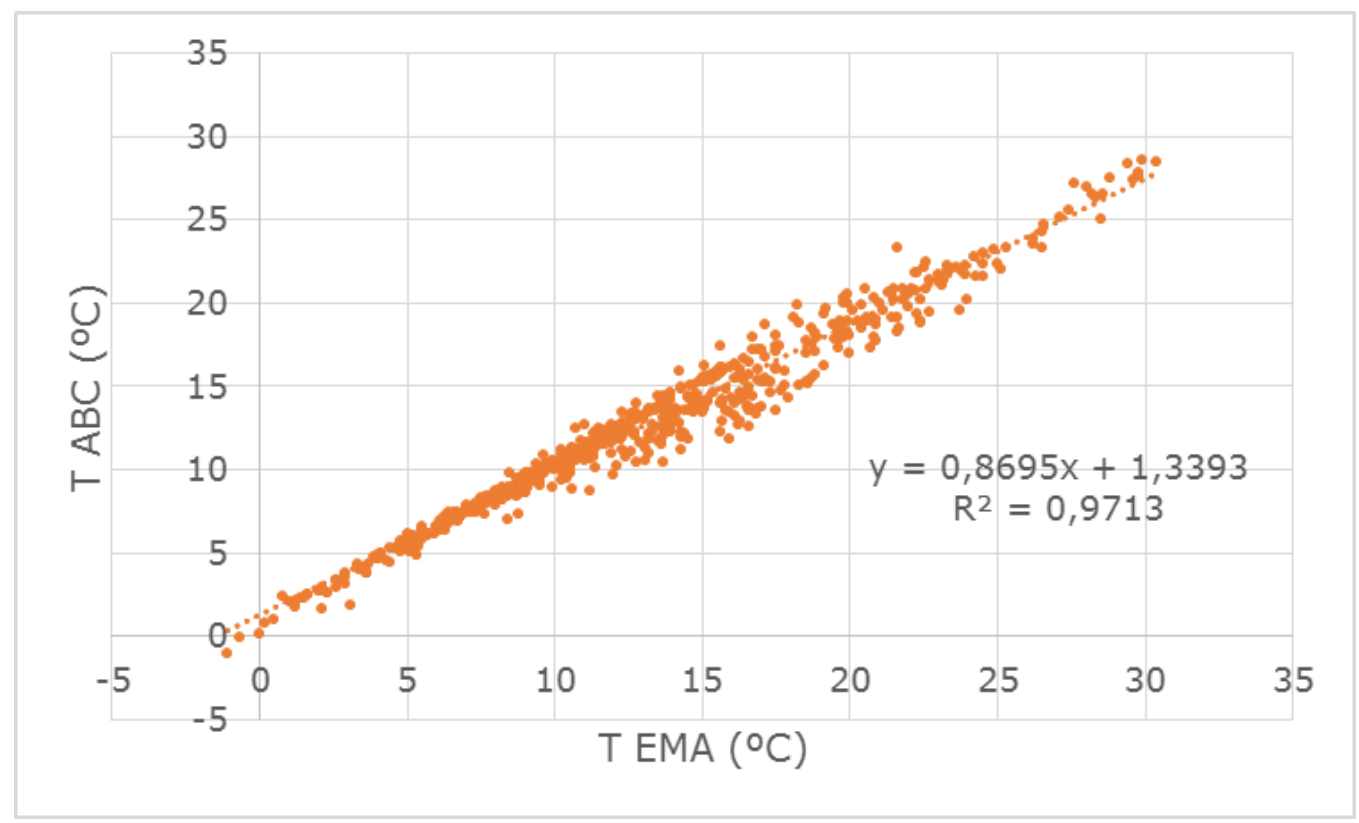


Figura 5 - Dispersão dos dados de temperatura do ar $\left({ }^{\circ} \mathrm{C}\right)$ do $\mathrm{T} A B C$ e da $\mathrm{T}$ EMA e coeficiente de correlação $\left(R^{2}\right)$ entre as duas variáveis.

\subsubsection{UMIDADE RELATIVA DO AR}

Em relação à umidade relativa do ar, observou-se que a amplitude higrométrica entre o abrigo meteorológico (UR abc) e a EMA (UR ema) foi de $1,7 \%$ (Tabela 7). Isso também está dentro do erro padrão estabelecido pelo fabricante, que é de $+/-3 \%$ para a umidade relativa do ar. Armani e Galvani (2006), ao compararem a umidade relativa do ar entre a do abrigo ABC e um abrigo padrão, observaram uma a variação de $1 \%$ entre os dois, o que fica dentro do erro do instrumento. Entretanto, Hoppe et al., (2015) encontraram uma diferença de $-8,5 \%$, evidenciando que os registros da umidade relativa do abrigo meteorológico de baixo custo são menores que os registrados no abrigo oficial.

Na EMA ocorreu o maior registro de umidade relativa máxima chegando bem próximo aos $100 \%$, enquanto a menor mínima com a umidade relativa do ar atingiu 31,1\%. Dessa maneira, a amplitude higrométrica mínima foi maior que a amplitude higrométrica máxima em comparação com os valores registrados em ambos os abrigos, onde a amplitude higrométrica máxima foi de $1,8 \%$.

A amplitude higrométrica da UR ABC é menor do que a amplitude da UR EMA, contrariando os resultados encontrados por Armani e Galvani (2006) em que a amplitude de UR ABC é maior do que o registrado no abrigo padrão. $O$ desvio entre os dados de umidade relativa do ar registrada pelos abrigos foi de $1,3 \%$. A variação da umidade relativa na EMA é mais elevada que no abrigo $A B C$ para ambos os equipamentos, ao contrário do que foi observado na temperatura do $\operatorname{ar}($ Tabela 3).

Tabela 3 - Parâmetros estatísticos elementares para a avaliação dos dados umidade relativa do ar (\%) para período de 01/08/2017 a 31/08/17.

\begin{tabular}{lccc}
\hline Parâmetros & UR ABC (\%) & UR EMA (\%) & UR ABC -UR EMA \\
\hline Média & 84,6 & 82,9 & 1,7 \\
\hline Mínimo & 39,2 & 31,1 & 8,1 \\
\hline Máximo & 97,7 & 99,5 & $-1,8$ \\
\hline Amplitude & 58,5 & 68,4 & $-9,9$ \\
\hline Desvio médio & 11,7 & 12,3 & $-0,6$ \\
\hline Desvio padrão & 14,6 & 15,9 & $-1,3$ \\
\hline
\end{tabular}

UR ABC: Umidade relativa do ar do abrigo meteorológico ABC;

UR EMA: Umidade relativa do ar da estação meteorológica;

UR ABC - UR EMA: Diferença entre a umidade do ABC e EMA.

Fonte: Trabalho de campo (2017).

Analisando-se a umidade relativa do ar para todo o período observou-se que a variação em ambos os abrigos foi similar. Nota-se que nos extremos, tanto a máxima quanto a mínima registradas no abrigo $A B C$ são elevadas. A umidade relativa do ar não ultrapassa os $99,5 \%$, entretanto a mínima é inferior a $40 \%$. Nessa perspectiva, a umidade relativa para os dois abrigos tem a maior variação entre 60 a 90\%, conforme se pode observar na Figura 6. 


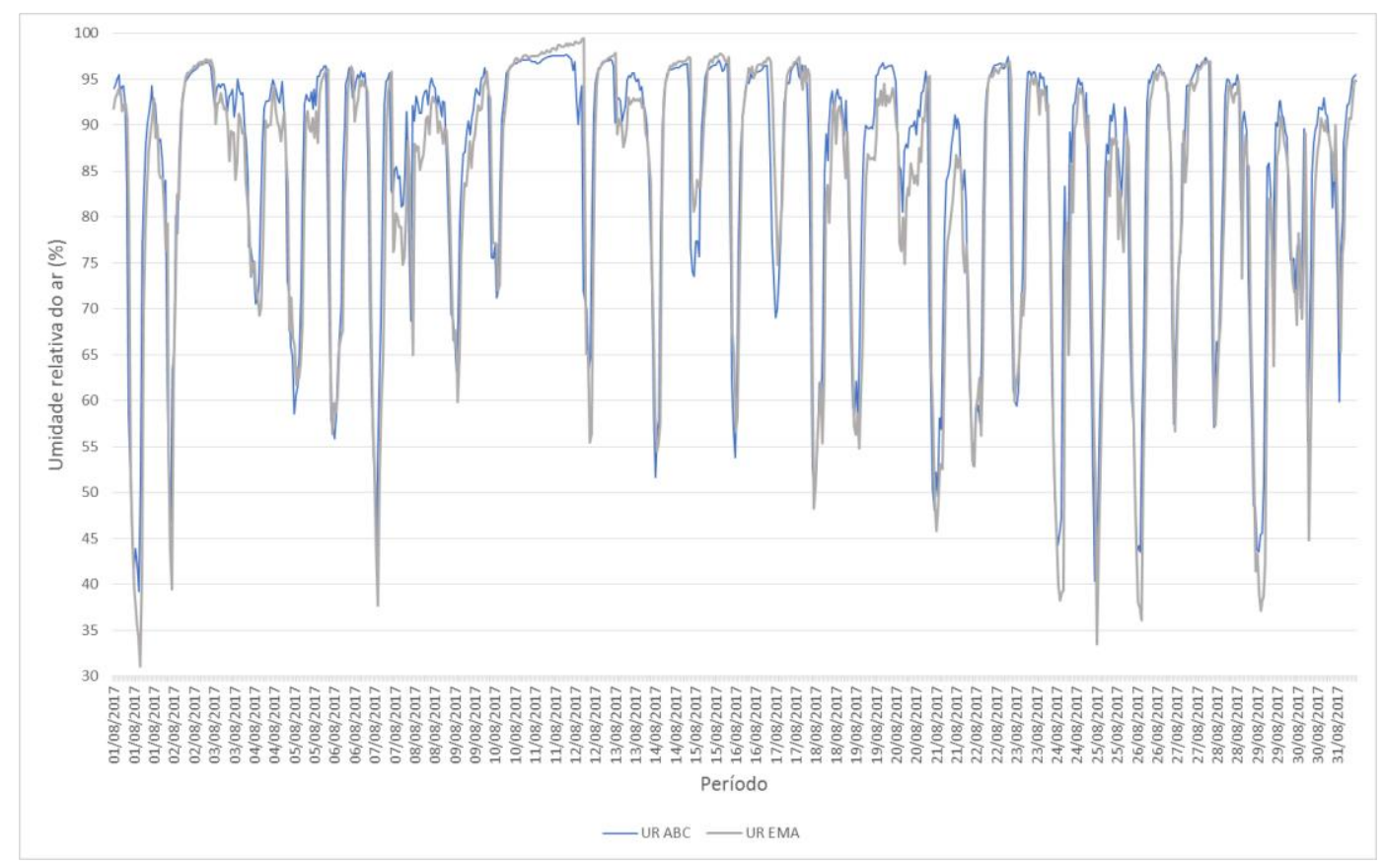

Figura 6 - Valores registrados para a média horária da umidade relativa do ar (\%) da EMA e do $A B C$ para o período analisado.

Ao analisar a média horária, observa-se que UR ABC começa a diminuir em torno das 7h e a UR EMA tem um atraso para começar a diminuir. Entre $12 \mathrm{~h} 00 \mathrm{~min}$ e $13 \mathrm{~h} 00 \mathrm{~min}$, a umidade relativa do ar em ambas permanece constante, sendo esse período o de máxima radiação solar. Conforme salienta Barry e Chorley (2003), a umidade relativa varia inversamente com a temperatura durante o dia, tendendo a ser mais reduzida no início da tarde e mais elevada no período noturno.

Dessa maneira, a partir das $14 \mathrm{~h} 00$ min no abrigo $A B C$ a umidade relativa do ar começa a aumentar com relação a umidade da EMA, que por sua vez começa a aumentar de maneira mais suave a partir das $15 \mathrm{~h} 00 \mathrm{~min}$. Armani e Galvani (2006) também salientam que a umidade relativa do ar no ABC diminui e aumenta mais rapidamente que a do abrigo padrão. A umidade ar média registrada na EMA tende a sempre ser mais baixa que a registrada no abrigo $A B C$, embora os valores extremos sejam registrados na EMA (Figura 7). 


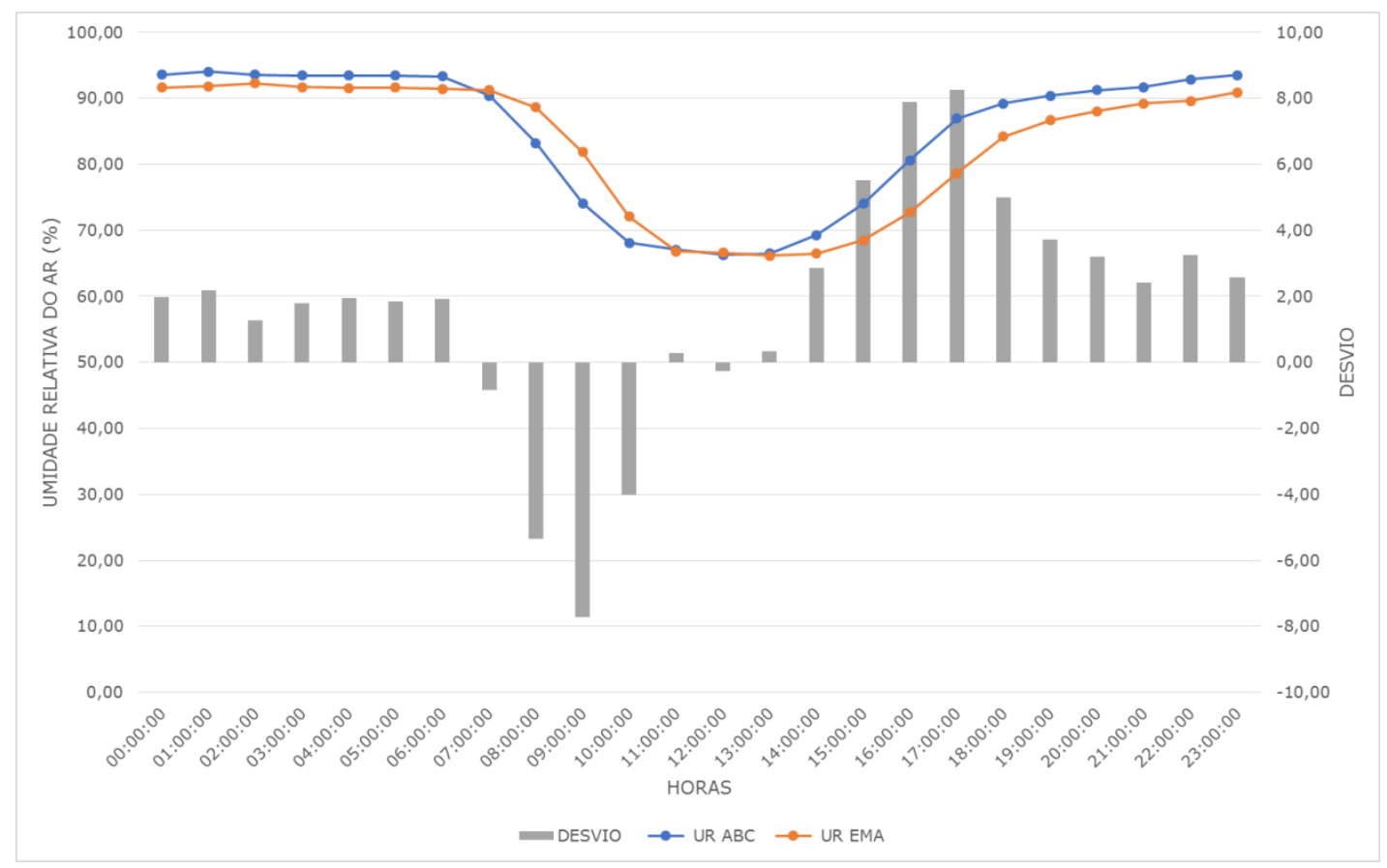

Figura 7 - Valores registrados para a média horária da umidade relativa do ar (\%) da EMA e do ABC para o mês de agosto de 2017.

Diante disso, na Figura 7 ainda observam-se os desvios registrados nos dois abrigos. Nas primeiras horas dos dias (00h00min até as 06h00min) permaneceram constantes, próximos ao 2,0\% Entretanto, no horário que começa o aquecimento e no de resfriamento o desvio se apresenta mais elevado, chegando até a $-8,0 \%$ no horário de aquecimento (09h00min) e 8,0\% às $17 \mathrm{~h} 00 \mathrm{~min}$.

O coeficiente de correlação entre os dois sensores para a umidade relativa do ar se mostrou eficiente, sendo de 0,90\%. O coeficiente de determinação também se mostrou satisfatório para os registros de umidade relativa do ar entre os dados do abrigo $A B C$ e da $\operatorname{EMA}(0,82)$, conforme a Figura 8. 


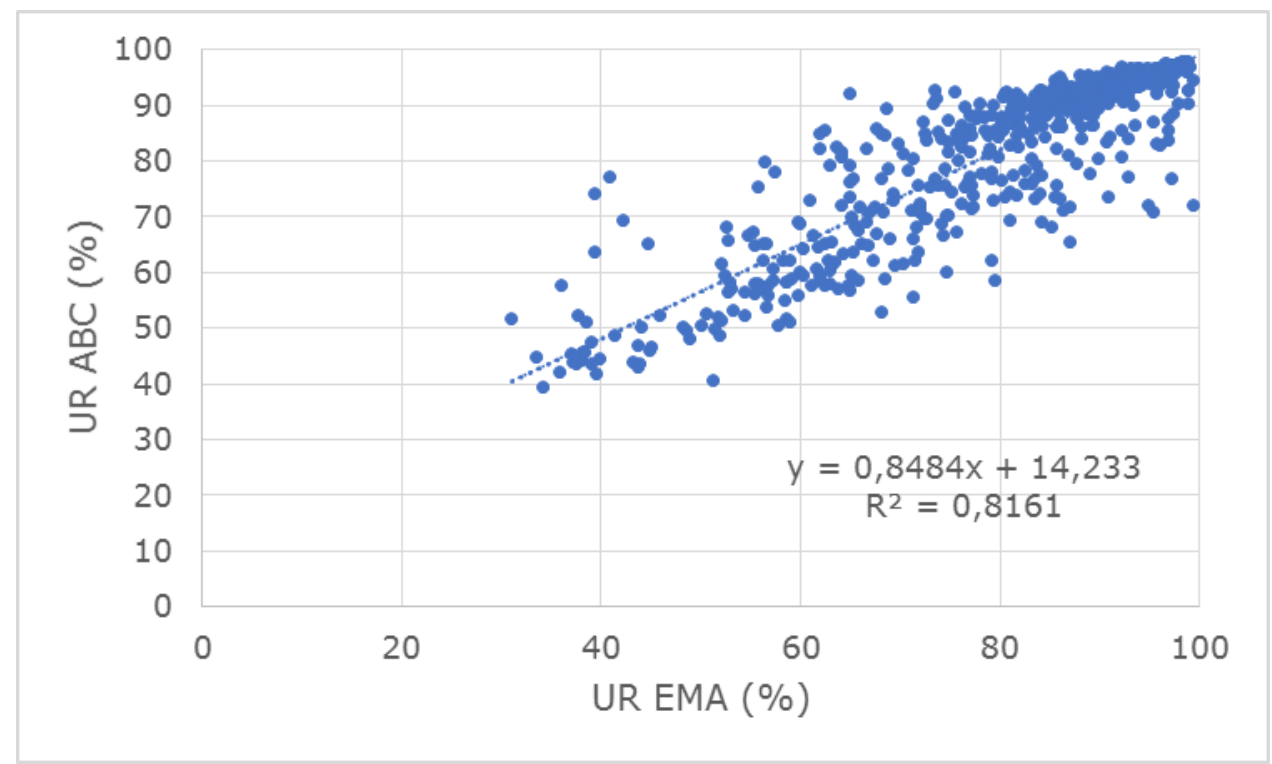

Figura 8 - Dispersão dos dados de umidade relativa do ar (\%) do abrigo ABC e da EMA e coeficiente de correlação $\left(R^{2}\right)$ entre as duas variáveis.

Por meio da análise e discussão dos resultados, pode-se concluir que o abrigo $A B C$ se mostrou eficiente para os dados de temperatura do ar e umidade relativa do ar. Isso mostra que os valores encontrados estão dentro do erro previsto pelo instrumento, ressaltado pelo fabricante. Ressalta-se que, em função da reduzida carga térmica do abrigo de baixo custo, ocorrem valores de temperatura do ar máxima e mínima que diferenciam do sensor padrão.

\section{CONSIDERAÇÕES FINAIS}

Diante do exposto observou-se que o abrigo $A B C$ se mostrou eficiente para os dados de temperatura do ar e umidade relativa do ar, sendo que 0 desvio para os dados de temperatura do ar foi de $0,3^{\circ} \mathrm{C}$ e $1,7 \%$ para a umidade relativa do ar. Ainda, os valores encontrados estão dentro do erro previsto pelos instrumentos, conforme indicado no manual do fabricante.

Diante disso, ressalta-se que em função da reduzida carga térmica do abrigo de baixo custo ocorrem valores de temperatura do ar máxima e mínima que diferenciam do sensor padrão. Com relação à umidade relativa do ar também são observados contrastes entre os valores registrados nos dois abrigos, nos períodos de aquecimento e resfriamento.

No entanto, o desempenho do abrigo $A B C$, quanto à temperatura e umidade relativa do ar para o período analisado e em condições de campo, se mostrou eficiente em relação aos dados da EMA. Assim, diferentemente dos outros abrigos apontados pela literatura, a calibração do abrigo desta pesquisa foi em condições de campo, o que proporciona a maior confiabilidade dos dados apresentados por ser um modelo já conhecido e testado por outros autores.

Assim, recomenda-se o uso desse tipo de abrigo (modelo $A B C$ ) para as pesquisas de campo de climatologia geográfica, em especial quando se tratar de pesquisas abaixo do dossel da vegetação e em locais de reduzida insolação. Outros modelos também recomentados pela literatura se destoam da pesquisa 
em função dos difíceis acessos dos locais de instalações. E muitas vezes, por serem equipamentos grandes, pesados ou que requerem energia elétrica e com custos elevados, não auxiliam no atual contexto da pesquisa climatológica brasileira.

\section{AGRADECIMENTOS}

O presente trabalho foi realizado com apoio do Programa Nacional de Cooperação Acadêmica da Coordenação de Aperfeiçoamento de Pessoal de Nível Superior - CAPES/Brasil - No Processo: 88881.068465/2014/01 no 071/2013 CAPES/PROCAD. A primeira autora agradece ao apoio do programa de bolsa de Demanda Social (DS-CAPES). O terceiro autor agradece o apoio do CNPq por meio da concessão da bolsa de pesquisa e produtividade (PQ1D).

\section{REFERÊNCIAS BIBLIOGRÁFICAS}

ALVES, R. R.; SERAFINI JÚNIOR, S. Miniabrigos Meteorológicos: Comparação e Análise Estatística Para Avaliação de Eficiência Nas Medições de Umidade Relativa do Ar. Anais...VX Simpósio Brasileiro de Geografia Física Aplicada, Vitória, 2013. p.526-535.

ARMANI, G.; GALVANI, E. Avaliação do desempenho de um abrigo meteorológico de baixo custo. Revista Brasileira de Agrometeorologia, Piracicaba-SP, v. 14, n.1, p. 116-122, 2006.

AZEVEDO, T. R. \& TARIFA, J. R. Miniabrigo meteorológico aspirado do Laboratório de Climatologia e Biogeografia e seu uso no estudo geográfico do clima. GEOUSP, São Paulo, n. 10, p. 165-174, 2001.

BARRY, G.; CHORLEY, R.J. Atmosphere, weather, and climate. 8th ed, Routledge, 2003. p.462.

BERNARD, J; KÉRAVEC, P; MORILLE, B; BOCHER, E; MUSY, M; CALMENT, I. Outdoor Air Temperature Measurement: A Semi-Empirical Model to Characterize Shelter Performance. Climate. 7, 26, 2019.

CASTELHANO, F. J.; ROSEGHINI.W.F.F, A utilização de policloreto de vinila (PVC) na construção de mini-abrigos meteorológicos para aplicação em campo, Revista Brasileira de Climatologia. N.9 p.48-55,2011

DOBRE, M; SESTAN, D; MERLONE, A. Air temperature measurement uncertainty associated to a mounting configuration temperature sensor-radiation shield. Disponível em:< https://www.wmo.int/pages/prog/www/IMOP/documents/>. Acesso em: 14 de ou. de 2019, 2018.

FIALHO, E. S; OLIVEIRA, W.D; ALOCCA, R de A; CORREA, W de S.C. Análise de desempenho de abrigos meteorológicos. Anais.... VIII Simpósio Brasileiro de Climatologia Geográfica, Juiz de fora, 2018, p. 1922-1933.

HOPPE I.L, et al. Comparação Entre Um Abrigo Meteorológico De Baixo Custo e a Estação Meteorológica Oficial Do Inmet em Santa Maria (RS). Anais... V Simpósio Internacional De Climatologia. 2014. 
HUBBARD, K. G.; LIN, X. "Air Temperature Errors Caused by Air Filter and Construction Effects on HMP45C Temperature Sensors in Weather Stations". Papers in Natural Resources. Vol. 46(2): 443-452, 2003.

GALVANI, E; LIMA, N. G. B; SERAFINI JUNIOR, S.; ALVES, R.R. Avaliação da temperatura do ar no perfil topoclimático do Parque Estadual Intervales - SP, entre as altitudes de 150 a 950 metros. In: SEMINÁRIO LATINO-AMERICANO DE GEOGRAFIA FÍSICA, 2008. Santa Maria. Anais...Santa Maria: UNIFRA e UFRGS.

GALVANI, E.; LIMA, N. G.B. a ocorrência de inversão térmica no perfil topoclimático do Pico das Agulhas Negras - RJ. In: VII Simpósio Brasileiro de Climatologia Geográfica, Anais... Rondonópolis, 2006. p.

GALVANI, E.; LIMA, N. G. B. de; FALCÃO, R. M. Ocorrência de Inversão Térmica no Perfil Topoclimático do Pico da Bandeira, Parque Nacional do Alto Caparaó, Brasil. IN: VI Seminário Latino Americano de Geografia Física. Anais... Coimbra: Universidade de Coimbra, 2010.

GOBO, J.P.A. Biocliamtologia subtropical e modelização do conforto humano: da escala local à regional. 2018, p. 396. Tese (Doutorado em Geografia FísicaPrograma de Pós-graduação em Geografia Física). Faculdade de Filosofia, Letras e Ciências Humanas da Universidade de São Paulo, São Paulo, 2018.

JARDIM, C.H. Construção e avaliação de desempenho de modelo de mini-abrigo meteorológico de ventilação passiva para experimentos em campo de climatologia. In: Emerson Galvani; João Paulo Assis Gobo; Nádia Gilma Beserra de Lima. (Org.). Climatologia Aplicada II. 1ed.Curitiba: CRV, 2018, v. 2, p. 221 232.

LIMA, N. G.B de; GALVANI, E.; FALCÃO, R.M. Análise da temperatura do ar no perfil topoclimático do pico da Bandeira, Parque Nacional Alto Caparaó, Brasil, entre as altitudes de 1106 m a 2892 m. IN: VI Seminário Latino-Americano de Geografia Física. Anais...Coimbra: Universidade de Coimbra, 2010.

LOPES, L. C. S. JARDIM, C. H. Variações de temperatura e umidade relativa do ar em área urbana e rural durante o segmento temporal de inverno de $2011 \mathrm{em}$ Contagem e Betim (MG). Acta Geográfica, p. 205-221, 2012.

MILANESI, M. A. Avaliação do Efeito Orográfico na Pluviometria nas Vertentes Opostas da Ilha de São Sebastião (Ilhabela- SP). 2007. 141 f. Dissertação (Mestrado em Geografia) - Faculdade de Filosofia, Letras e Ciências Humanas da Universidade de São Paulo, São Paulo, 2007.

ROJAS, J; GILETE, S.D; MARZON, J. Development of a Low-Cost Weather Station to Measure in Situ Essential Climate Variables. Journal of Earth Science and Engineering, 455-463p., 2014.

SANTOS, D.D dos; MORAES, S. L de; GALVANI, E. Variação da temperatura do ar média, mínima e máxima no perfil topoclimático da Trilha Caminhos do Mar (SP). Revista Equador: Teresina, PI. Vol. 5, No 5, 2015. p.01 - 19.

SERAFINI JÚNIOR, S.; ALVES, R. R. Miniabrigos meteorológicos: comparação e análise estatística para avaliação de eficiência. GEOUSP - Espaço e Tempo (Online), São Paulo, v. 18, n. 1, 2014, p. 198-210.

SILVA, M.S.D; SANCHES, R.G; SANTOS, B.S dos; VECCHIA, F. A da S. Desenvolvimento de um termohigrômetro de baixo custo para estudos 
climáticos e ambientais. Anais.... VIII Simpósio Brasileiro de Climatologia Geográfica, Juiz de fora, 2018, p. 1783-1802.

SIMIONI, J. P.D; WOLLLMANN, C. A. VARIABILIDADE ESPACIAL, DOS ATRIBUTOS CLIMÁTICOS NA ESTAÇÃO ECOLÓGICA DO TAIM, RS: ANÁLISE DURANTE O OUTONO E INVERNO DE 2013. Geografia Ensino \& Pesquisa, v. 18, p. $135-148,2015$.

SIMIONI, J. P. D; WOLLLMANN, C. A. Variabilidade sazonal dos atributos climáticos e definição das unidades climáticas na estação ecológica do Taim, RS. GEOUSP: espaço e tempo, v. 20, p. 676-697, 2016.

VALIN JÚNIOR, $M$ de, O; SANTOS, F. M de M; NOGUEIRA, M.D. de J. A; MUSIS, C.R de; NOGUEIRA, J. de $S$. Utilização de abrigos termo-higrométricos alternativos. CAMINHOS DE GEOGRAFIA - revista online. Uberlândia v. 17, n. 57 Mar/2016 p. 74-91.

TARARA, J. M.; HOHEISEL, GWEN-ALYN. Low-cost Shielding to Minimize Radiation Errors of Temperature Sensors in the Field. Hortscience 42(6):13721379p., 2007.

TORRES, J. D.; MONTEIRO, I. O.; SANTOS, J. R.; ORTIZ, M. S.; Aquisição de dados meteorológicos através da plataforma Arduino: construção baixo custo e análise de dados, Scientia Plena 11, 021712, 2015.

WOLLMANN, C. A; SIMIONI, J. P. D. VARIABILIDADE ESPACIAL DOS ATRIBUTOS CLIMÁTICOS NA ESTAÇÃO ECOLÓGICA DO TAIM (RS), SOB DOMÍNIO POLAR. Revista do Departamento de Geografia, v. 25, p. 56-76, 2013.

WOLLLMANN, C. A ; SIMIONI, J. P. D; IENSSE, A. C. Atlas Climático do Taim: contribuição ao estudo do clima em unidades de conservação. Boletim Geográfico do Rio Grande do Sul, v. 1, p. 30-50, 2016.

WMO, WORLD METEOROLOGICAL ORGANIZATION. Guide to Meteorological Instruments and Methods of Observation. n. 8, 2010.

YANG, J; LIU, Q.; DAI, W.; DING, R. Fluid dynamic design and experimental study of an aspirated temperature measurement platform used in climate observation. Review of scientific instruments, n. 87, 2016. 